Article

\title{
Environmental pollution and breast cancer: the microplastic component BPA regulates the intratumoral immune microenvironment and increases lung metastasis
}

\author{
Margarita Isabel Palacios-Arreola1 ${ }^{1}$, Karen Elizabeth Nava-Castro', Norma Angelica Mendoza-Moreno ${ }^{2}$, Mariana \\ Segovia-Mendoza ${ }^{3}$, Armando Perez-Torres ${ }^{4}$ and Jorge Morales-Montor ${ }^{5 *}$ \\ 1 Laboratorio de Genotoxicología y Medicina Ambientales. Departamento de Ciencias Ambientales. Centro \\ de Ciencias de la Atmósfera. Universidad Nacional Autónoma de México. 04510. Ciudad de Mexico, \\ México; mi.palacios.arreola@gmail.com \\ 2 Departamento de Biologìa Celular, Instituto de Investigaciones Biomédicas, Universidad Nacional \\ Autónoma de México, AP 70228, Ciudad de México 04510, México; angelica@correo.biomedicas.unam.mx. \\ 3 Departamento de Farmacología, Facultad de Medicina, Universidad Nacional Autónoma de México México, \\ 045100. Ciudad de México; mariana.segovia@facmed.unam.mx \\ 4 Departamento de Biologia Celular y Tisular, Facultad de Medicina, Universidad Nacional Autónoma de \\ México, Edificio A, 4to piso, Ciudad Universitaria, México DF, 04510, Mexico. armandop@unam.mx \\ 5 Departamento de Inmunología, Instituto de Investigaciones Biomédicas, Universidad Nacional Autónoma \\ de México, AP 70228, Ciudad de México 04510, México; jmontor66@biomedicas.unam.mx \\ * Correspondence: Jorge Morales Montor, email: jmontor66@biomedicas.unam.mx, Phone: +52555-56223158, \\ Fax: $+52555-56223369$
}

Simple Summary: The widely spread microplastic component and endocrine disruptor, BPA, it is a hazardous material recognized for long time. In here, for the first time, it is demonstrated that BPA, administered into mice in a very specific developmental step of the animal (3 days post-natal), induces in the adult life an increase in metastasis to the lung, compared to control and vehicle mice. Also, of novelty it is the analysis of the cytokine tumor microenvironment, which it is the reason of the increased metastasis by BPA (BPA induce an increase in pro-metastatic cytokines)

\begin{abstract}
Background: Metastasis is a complex process that involves the spread of the tumor to distant parts of the body from its original site. Metastatic dissemination represents the main physiopathology of cancer. Soluble factors such as cytokines have been closely related to breast cancer (BC) metastasis. Bisphenol A (BPA) is an endocrine disrupting chemical compound with estrogenic properties, which exposure in the early stages of neonatal life leads to an increase in the size and weight of breast tumors and cellular changes in the tumoral immune microenvironment. Methods: Thus, we used female BALB/c mice that were exposed neonatally to a single dose of BPA. Once sexual maturity was reached, a mammary tumor was induced injecting $4 \mathrm{~T} 1$ cells in situ. After 25 days of injection, we evaluated endocrine alterations, cytokine expression, tissue alterations denoted by macro and micro metastases in the lung, and metastasis-induced cell infiltration. Results: BPA neonatal treatment did not show significant endocrine alterations. Nevertheless, BPA induced a great rate of metastasis to the lung associated with higher intratumoral expression of IL1b, IL-6, IFN-g, TNF-a and VEGF. Conclusions Our data suggest that cytokines are key players in $\mathrm{BC}$ metastasis induction, and that BPA is a risk factor to be considered. This knowledge must be considered with the aim of recognizing environmental pollution in the clinical history of patients to possibly counter BC metastases.
\end{abstract}

Keywords: Breast cancer; metastasis; cytokines; microenvironment; bisphenol A; endocrine disruptors 


\section{Introduction}

$\mathrm{BC}$ is among the most prevalent types of cancer worldwide [1]. Breast tumors can be generated in different areas in the breast such as lobules, ducts, and connective tissue [2] About $80 \%$ of all breast cancers are invasive ductal carcinomas [3]. However, the hallmark of cancer pathology is not the tumor itself, but the migration of transformed cells to a different tissue of the tumor origin, a process widely known as metastasis [4]. In this biological mechanism, immunological factors, particularly cytokines, play a fundamental role [5]. In addition, several environmental factors have been linked to an increased incidence of $\mathrm{BC}$ and the promotion of metastasis [6, 7]. Environmental pollution has gained a lot of attention related to BC. Exposure to chemicals from different sources has now been associated with the risk of developing BC and a much more aggressive form when the tumor is established (higher risk of metastasis) [8, 9].

When talking about cancer, responsible mechanisms that contribute to the timely diagnosis for the detection and elimination of malignant cells must be taken also into account. In this context, cellular and soluble factors of the immune system play a major role in preventing cancer development or controlling its progression [10,11]. However, in the tumor microenvironment, these factors can also contribute to tumor progression.

On the other hand, metastasis is a complex process that involves tumor spread to distant parts of the body from its original site. To successfully colonize a distant organ in the organism, a cancer cell must complete a series of steps before it becomes a clinically detectable lesion. Cancer cells use two main dissemination pathways: the lymphatic pathway, leading to the invasion through lymph nodes draining the organs where the tumor evolves; and the blood pathway, which leads to the invasion of distant organs. In $\mathrm{BC}$, metastasis is preferentially targeted to the bones, lung, liver, and brain [12-14]. Regarding the above, a very important condition that favors BC metastasis is angiogenesis, by which the tumor promotes the formation of abnormal vasculature to provide the tumor with oxygen and nutrients. Among proangiogenic factors are cytokines such as the endothelial growth factor receptor (VEGF) [15, 16].

On the other hand, human activity has led to an increase in the number of environmental pollutants, including endocrine disrupting chemicals (EDCs). BPA is widely used as a monomer in the production of polycarbonate plastics, epoxy resins, and dental sealants [17]. This compound can be easily released from these materials due to incomplete polymerization or hydrolysis of the polymers that contain it, which can occur when exposed to high temperatures, acidic conditions, or enzymatic processes [18]. The main source of exposure to BPA in animals and humans is in food and beverages that have been in contact with materials manufactured with BPA, which is detached from its matrix and is ingested by different routes [19]. BPA is classified as an EDC with estrogenic character, as it can bind to estrogen receptors, triggering signaling pathways, even when its affinity is lower than the endogenous ligand, 17 $\beta$-estradiol [20]. Despite the Food and Drug Administration (FDA) and the European Food Safety Agency (EFSA) calculated that the Tolerable Daily Intake of BPA is $50 \mu \mathrm{g} / \mathrm{kg} /$ day, it has been estimated that exposure to BPA per food package was higher in children from 1 - 2 months of age [21]. In addition, exposure to BPA at tolerable concentrations or below has been shown to be related to negative effects on the health of humans and rodents $[9,22]$. The nature and magnitude of BPA adverse effects depend on the dose, the course of exposure, and the developmental stage in which exposure occurs. It is well known that the exposure of BPA can occur as early as during gestation, as evidenced by reports of BPA presence in amniotic fluid, fetal serum, and breast milk [23-25]. In this regard, there is an existing concern about the effects that BPA could exert on a developing organism, including the immune system [26-28]. Previously, we have shown that after 25 days of injection, without major endocrine alterations, mice exposed to BPA developed larger tumors, animals showing a higher proportion of regulatory $\mathrm{T}$ lymphocytes in the BPA exposed group, together with decreased expression of TNF- $\alpha$ and IFN- $\gamma$, as well as the M2 macrophage marker Fizz-1 in the BPA exposed group. Furthermore, in the workflow, cytometric analysis revealed 
differences in the expression of estrogen receptor (ER) $\alpha$ in T lymphocytes, macrophages, and NK cells, both associated with exposure to BPA and tumor development [29].

Therefore, we decided to assess whether exposure to BPA in a critical development period affects not only tumor size, but also lung metastasis and cytokine expression pattern in tumors. Our results demonstrated that BPA administered during the neonatal period evoked an increase on lung metastasis and intratumoral cytokine proinflammatory pattern during the adult life.

\section{Materials and Methods}

\subsection{Ethics statement}

Animal care and experimental practice were conducted at the Unidad de Modelos Biológicos (UMB) in the Instituto de Investigaciones Biomédicas (IIB), Universidad Nacional Autónoma de México. All experimental procedures in the animals were approved by the Institutional Care and Animal Use Committee (CICUAL), permit number 155, adhering to Mexican regulation (NOM-062-ZOO-1999), and in accordance with the recommendations from the National Institute of Health (NIH) of the United States of America (Guide for the Care and Use of Laboratory Animals). Euthanasia of experimental animals was performed humanely by overdose of inhaled sevofluorane (Abbot, México).

\subsection{Animals}

Mice of the syngeneic strain BALB/c AnN (H2-d) were purchased from Harlan México (Facultad de Química, UNAM, México). The animals were housed at UMB with controlled temperature $\left(22^{\circ} \mathrm{C}\right)$ and 12-hrs light-dark cycles, with water and Purina LabDiet 5015 (Purina, St. Louis MO) chow ad libitum. After neonatal treatment, only female mice were used for experimentation.

\subsection{Neonatal BPA exposure}

Although the main route of exposure to BPA is commonly oral, subcutaneous injection was selected instead, as no differences between oral and subcutaneous routes are observed in neonate mice in this case. To resemble the human final gestational stage and aiming at the murine critical immune system development ( $T$ lymphocytes developmental window) mice were exposed at postnatal day 3 (PND3). Briefly, 72 hours after birth female pups were identified by ano-genital distance. Only female pups received treatment, although entire litters were assigned to experimental groups to avoid pup reallocation stress. The intact group received no neonatal treatment. The vehicle group received a dorsal subcutaneous injection of $20 \mu \mathrm{l}$ of corn oil as the vehicle (Sigma, St. Louis $\mathrm{MO}$ ). The BPA group received $250 \mu \mathrm{g} / \mathrm{kg}$ body weight (bw) of BPA, dissolved in corn oil. Given that neonate rodents have minimal glucuronidation activity, which is the major metabolic mechanism for BPA clearance, this dose approximates to a brief, 5 days exposure to the FDA reference dose of $50 \mu \mathrm{g} / \mathrm{kg}$ bw/day, but performed in a single administration, thus avoiding excessive manipulation stress. The pups were weaned at 21 days of age and placed in standard cages, 5 mice per cage.

\subsection{Assessment of endocrine parameters}

Vaginal opening. From 25 days old forth, the vaginal opening was examined by holding the mice in a dorsal restraint and using a light extension of the peri-vaginal skin. Estrous cycle. At 8 weeks old, the oestrous cycle was assessed using a vaginal smear wash of $50 \mu \mathrm{l}$ saline solution (PiSA, Guadalajara México), followed by Giemsa stain and light microscope observation. Serum samples, corresponding to the diestrus phase, were used to determine estradiol levels, using the EIA DetectX® Serum 17 $\beta$-estradiol kit (Arbor Assays, Ann Arbor MI), according to the manufacturer's protocol. 


\subsection{Cell culture}

The 4T1 cell line (ATCC® CRL-2539) was kindly donated by Dr. Pedro Ostoa-Saloma and cultivated in RMPI 1640 medium (Sigma, St. Louis MO) supplemented with 10\% FBS (ByProductos, Guadalajara México). Subculture was performed at 70-80\% confluency. After a second subculture, cells were harvested and resuspended in $0.9 \%$ saline at a concentration of 250,000 cells $/ \mathrm{ml}$ for inoculation.

\subsection{Mammary tumor induction}

Upon sexual maturity ( 8 weeks old), mice from every exposure group were randomized into secondary experimental groups, ie, control (without tumor induction) and 4T1 (tumor induction) groups. Mice assigned to 4T1 groups were treated as follows: Mice were anesthetized by inhalation of a mixture of air and $5 \%$ sevofluorane. After low abdomen asepsis, the fourth nipple was located and $10^{4} 4 \mathrm{~T} 1$ cells were introduced by a single injection into the mammary fat pad. Tumor growth was monitored for 25 days.

\subsection{Histological analysis of lungs}

The lung samples obtained from the experimental animals were fixed in $4 \%$ paraformaldehyde (J.T. Baker, México), dehydrated, and embedded in paraffin. Nonserial, longitudinal tissue blocks were cut into $4 \mu \mathrm{m}$ thick sections and mounted on polyL-lysine coated slides (Sigma, St Louis, MO, USA). The histological analyses were performed with hematoxylin-eosin staining to identify neutrophils. The number of each cell type in the lung was calculated using a 40X objective. Several microscope fields, equivalent to $1 \mathrm{~mm} 2$, were analyzed for each mouse. The empty areas within the tissue were discarded using the software Image J. A total area of $1 \mathrm{~mm} 2$ of lung was analyzed per group. The identification criteria were based on the morphological characteristics of the cells, which were also quantified according to each type.

\subsection{Tumor cytokine immunofluorescence}

Tumors from all groups were fixed in $4 \%$ paraformaldehyde for $48 \mathrm{~h}$, washed with PBS, and stored in PBS containing 30\% sucrose at $4{ }^{\circ} \mathrm{C}$ overnight. The next day, the samples were embedded in tissue freezing medium (Leica, Nussloch, Germany) and frozen at $-70^{\circ} \mathrm{C}$ (dry ice hexane bath). Serial sections of $20 \mu \mathrm{m}$ thickness were obtained using a cryomicrotome, placed on slides coated with poly-L-lysine (Sigma) and air-dried. The sections were treated with $1 \%$ Triton X-100, blocked with $1 \%$ albumin (BSA) and incubated overnight at $4{ }^{\circ} \mathrm{C}$ with primary antibody diluted in 1:1000 BSA-PBS. Antimouse IL-1 $\beta$, IL-4 and VEGF performed in rabbit, anti-mouse IFN- $\gamma$, TNF- $\alpha$, IL-10, and IL-6 performed in goat were used as primary antibodies (Santa Cruz, Bio-technology, USA). Anti-rabbit IgG and anti-goat IgG conjugated with rhodamine (TRITC) were used as secondary antibodies (ZIMED Laboratories Inc., USA). After rinsing in PBS, sections were incubated with the secondary antibody for $1 \mathrm{~h}$ at room temperature diluted in 1:200 BSA-PBS, washed in PBS, and embedded in anti-fading DAKO mounting medium (DAKO, USA). Tumor sections processed without the primary antibody were used as negative controls.

\subsection{Statistical analysis}

The general experimental design considers 2 independent variables: neonatal exposure (Intact, Vehicle of BPA) and mammary tumor induction (Control or 4T1). The data regarding tumor development and tumor microenvironment only considers the exposure variable, as all animals belong to 4T1 group. Data from 2-3 independent experiments were analyzed with Prism 6® software (GraphPad Software Inc.) and charted as mean \pm standard deviation. Data distribution normality was assessed via Shapiro-Wilk test. Thereafter, a one-way ANOVA $(p=0.05)$ was performed, followed by a Tukey posthoc test. Differences were considered significant when $p<0.05$, with the actual $p$ value being stated in each figure legend. The data regarding cytokine expression considers both 
independent variables and therefore, a two-way ANOVA $(\alpha=0.05)$ was performed, followed by a Holm-Sidak post-hoc test, with the same significant difference criterion.

\section{Results}

\subsection{Endocrine Parameters}

The experimental strategy we employed involves the development of a tumor within a hormone-sensitive tissue; therefore, it was important to discriminate whether the observed effects could be attributed to the direct or indirect effect of neonatal endocrine disruption on the immune system or caused by persistent hormonal alteration. To assess the potential reproductive effects of a single $250 \mu \mathrm{g} / \mathrm{kg}$ bw BPA dose, puberty onset was determined by the age of vaginal opening. We observed that exposure of female mice to BPA did not alter puberty onset. Furthermore, BPA did not influence baseline serum levels of estradiol during the diestrus phase (Table 1).

Table 1. Endocrine parameters assessment in female mice, the vaginal opening as puberty onset indicator.

\begin{tabular}{|c|c|c|c|c|}
\hline Treatment & $\begin{array}{c}\text { Vaginal } \\
\text { Opening (Days) }\end{array}$ & Serum $17 \beta$ estradiol (pg/ml) & $\begin{array}{c}\text { Complete } \\
\text { Estrous Cycle }\end{array}$ & $\begin{array}{c}\text { Tumor weight } \\
\text { (grams) }\end{array}$ \\
\hline Control & 30 & $64 \pm 3$ & Yes & NO TUMOR \\
\hline Vehicle & 30 & $63 \pm 4$ & Yes & NO TUMOR \\
\hline BPA & 30 & $62 \pm 5$ & Yes & NO TUMOR \\
\hline Control 4T1 & 30 & $56 \pm 9$ & No & $0.9 \pm .1$ \\
\hline Vehicle 4T1 & 30 & $63 \pm 5$ & No & $1.2 \pm .3$ \\
\hline BPA $4 \mathrm{~T} 1$ & 30 & $65 \pm 1$ & No & $2.1 \pm .25$ \\
\hline
\end{tabular}

\subsection{Tumour size and weight}

A remarkable promotion of tumour development was observed in the BPA-exposed group. At 25 days after the inoculation of tumour cells, it was evident that the mice subjected to neonatal BPA exposure developed greater tumours (Table 1). In fact, we confirm and extend our previous findings that after measuring tumor weight, those found in mice exposed to BPA showed a 2.4-fold increase in weight compared to the unexposed and vehicle groups (Table 1).

\subsection{IL-1 $\beta$ intratumoral expression pattern}

Figure 1A shows a representative set of immunofluorescence images that belong to intratumoral expression of IL-1 $\beta$ in the different experimental groups: control, vehicle, and BPA. Figure 1B shows the quantification of IL-1 $\beta$ expressed by the mean fluorescence intensity of the previous images. The results demonstrated that the expression of IL- $1 \beta$ was greater in the BPA group as compared to the control and vehicle treatments. 
A
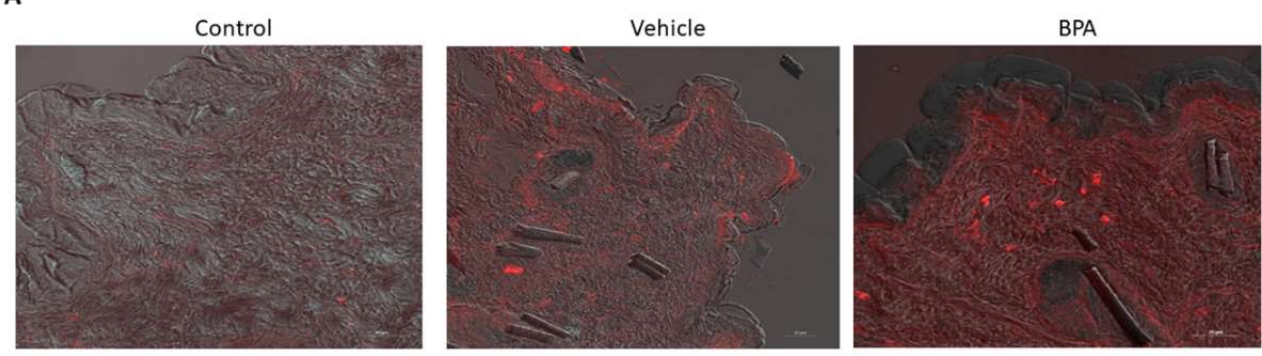

B

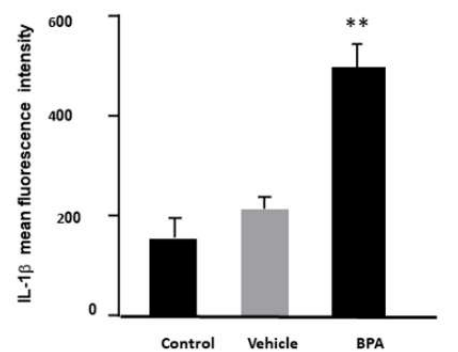

Figure 1. Intratumoral expression of IL-1 $\beta$. A. Representative images belonging to the intratumoral expression of IL-1 $\beta$ corresponding to the three experimental groups; control, vehicle, and BPA. B. Quantification of the expression of IL-1 $\beta$. Each bar epresents the mean of a representative three different experiments ${ }^{* *}$ significant differences vs control and vehicle groups.

\subsection{IL-4 intratumoral expression pattern}

IL-4 expression was also performed. Again, Figure 2A contains representative images of the immunofluorescence stains corresponding to the expression of this cytokine in the different experimental groups. When IL-4 was quantified (Figure 2B), the tumors belonging to the control and vehicle groups showed a higher expression of IL-4 compared to tumors from animals treated with $\mathrm{BPA}$, in which there is a clear decrease in its expression.

A
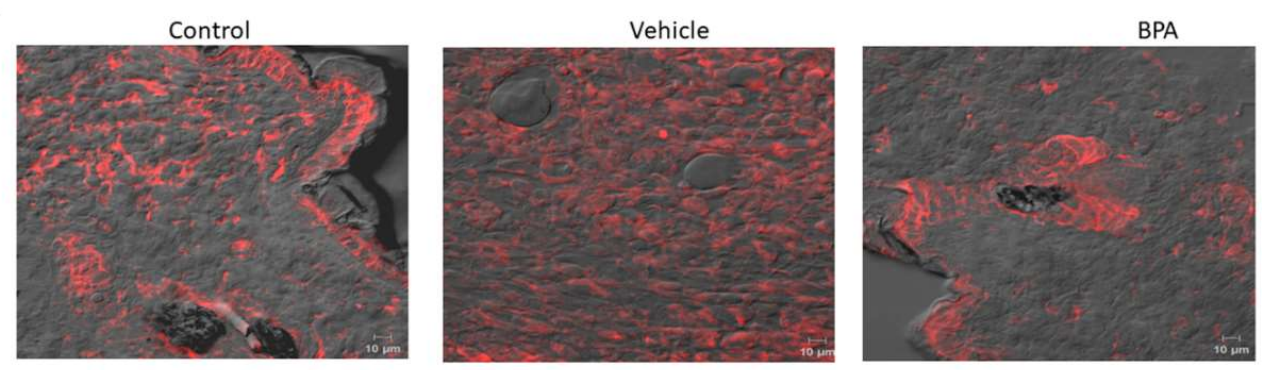

B

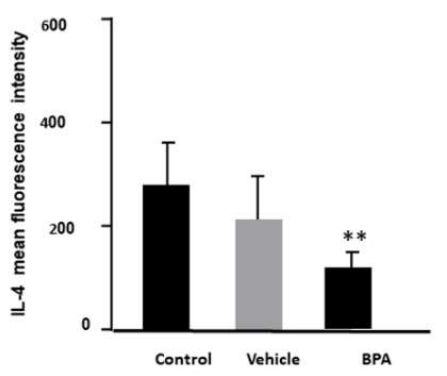

Figure 2. Intratumoral expression of IL-4. A. Representative images belonging to the intratumoral expression of IL-4 corresponding to the three experimental groups; control, vehicle, and BPA. B. Quantification of the expression of IL-4. Each bar epresents the mean of a representative three different experiments ${ }^{* *}$ significant differences vs control and vehicle groups. 


\subsection{IL-6 intratumoral expression pattern}

The results on IL-6 expression are present in Figure 3. The expression of this molecule was strong in the control and vehicle groups as denoted by the high fluorescence intensity, while in the BPA group, there was a significantly higher expression of IL-6. It is noteworthy that fluorescence quantification produced a 2.9 increase in BPA-induced intratumoral levels of IL-6 compared to the control and vehicle groups (Figure 3B).

A
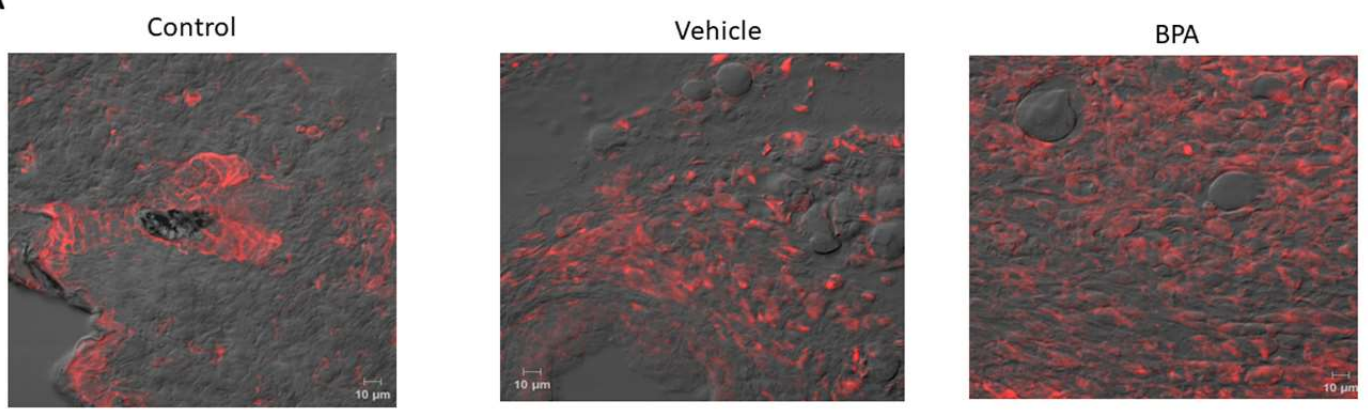

B

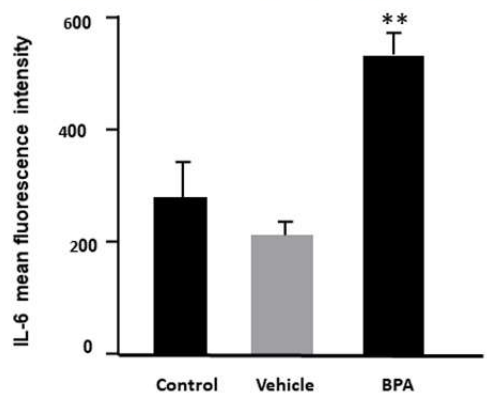

Figure 3. Intratumoral expression of IL-6. A. Representative images belonging to the intratumoral expression of IL-6 corresponding to the three experimental groups; control, vehicle, and BPA. B. Quantification of the expression of IL-6 Each bar epresents the mean of a representative three different experiments ** significant differences vs control and vehicle groups.

\subsection{IL-10 intratumoral expression pattern}

The immunoregulatory cytokine IL-10 was also searched in the tumors of all experimental groups. Intact and vehicle groups developed a low fluorescence of IL-10. On contrary, increased levels of fluorescence were found in the tumors of the BPA-treated mice (Figure 4A). Regarding the quantification of IL-10 (Figure 4B) comparison between the expression of control and vehicle, with BPA, showed a 2-fold increase in the expression of IL-10 (Figure 4B). 


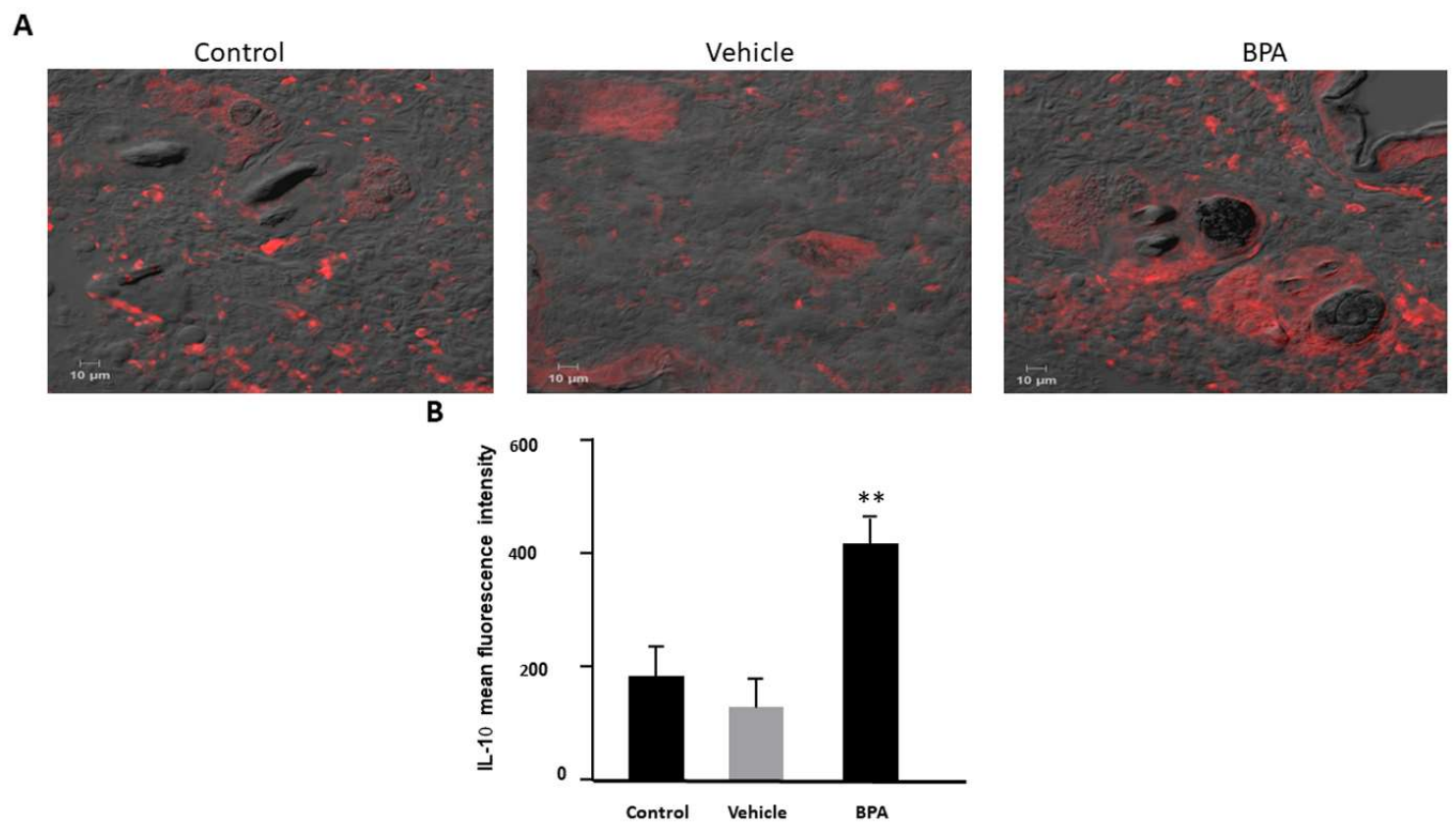

Figure 4. Intratumoral expression of IL-10. A. Representative images belonging to the intratumoral expression of IL-10 corresponding to the three experimental groups; control, vehicle, and BPA. B. Quantification of the expression of IL-10 Each bar epresents the mean of a representative three different experiments ${ }^{* *}$ significant differences vs control and vehicle groups.

\subsection{TNFo intratumoral expression pattern}

Intact and vehicle groups developed a mild fluorescence of TNF-a, with increased levels of fluorescence in the tumors of the BPA-treated mice (Figure 5A). As for the quantification of TNF- $\alpha$ (Figure 5B) comparison between the expression of control and vehicle, with BPA, showed a 3-fold increase in TNF-a expression (Figure 5B). 
A
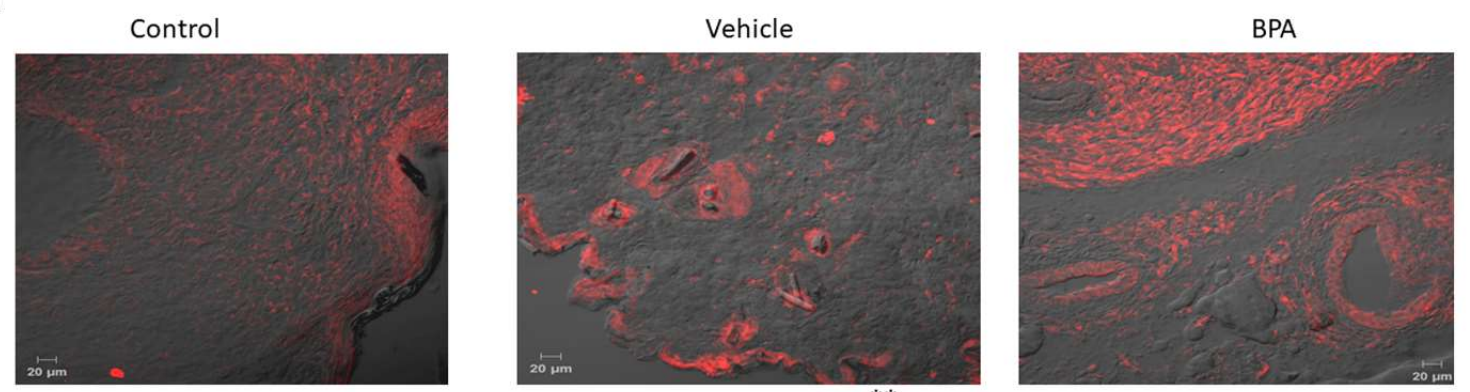

B

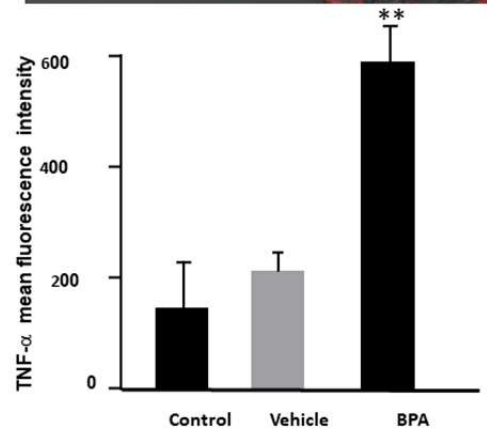

Figure 5. Intratumoral expression of TNF $\alpha$. A. Representative images belonging to the intratumoral expression of TNF $\alpha$ corresponding to the three experimental groups; control, vehicle, and BPA. B. Quantification of the expression of TNF $\alpha$ Each bar epresents the mean of a representative three different experiments ${ }^{* *}$ significant differences vs control and vehicle groups.

\subsection{IFN- $\gamma$ intratumoral expression pattern}

Regarding intratumoral IFN-g expression (Figure 6), the BPA-treated group showed higher levels of fluorescence compared to the control and vehicle groups, which showed mild levels of fluorescence (Figure 6A).. When fluorescence levels were quantified (Figure 6B), there was a significant 1.7-fold increase in IFN- $\gamma$ intratumoral expression of IFN- in the BPA group, compared to the control and vehicle groups. 
A
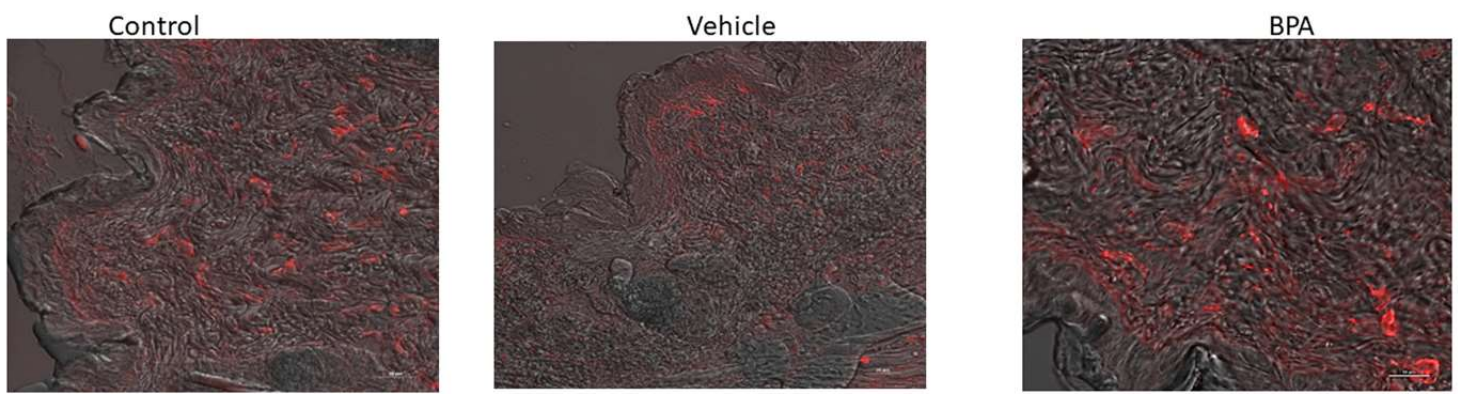

B

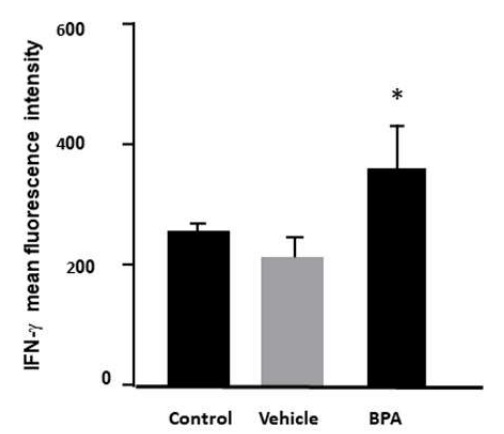

Figure 6. Intratumoral expression of IFN $\gamma$. A. Representative images belonging to the intratumoral expression of IFN $\gamma$ corresponding to the three experimental groups; control, vehicle, and BPA. B. Quantification of the expression of IFN $\gamma$ Each bar epresents the mean of a representative three different experiments * significant differences vs control and vehicle groups.

\subsection{VEGF intratumoral expression pattern}

The expression of VEGF was also evaluated (Figure 7). Interestigly tumors exposure to BPA had higher levels of fluorescence as compared with the control and vehicle groups (Figure 7A). It is noteworthy that the presence of this cytokine was distributed along the evaluated tumor court. Visual evaluation of VEGF was confirmed when quantification was performed (Figure 7B), and BPA-treated animals developed a 3-fold increase in VGEF expression, compared to the control and vehicle groups (Figure 7B). Notably, the different negative controls of each cytokine evaluated are compiled in Supplementary Figure 1. 
A

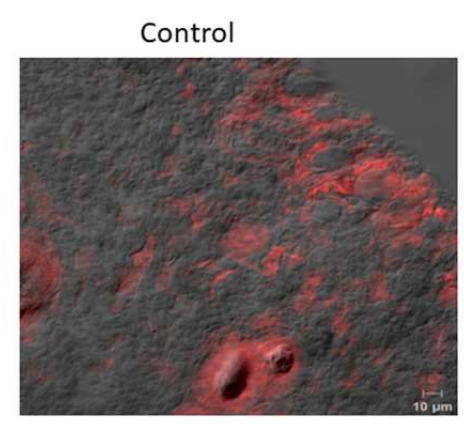

B

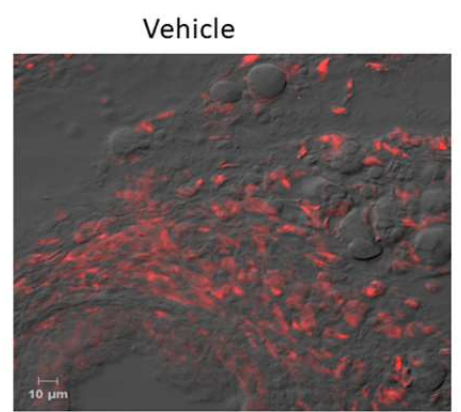

$* *$
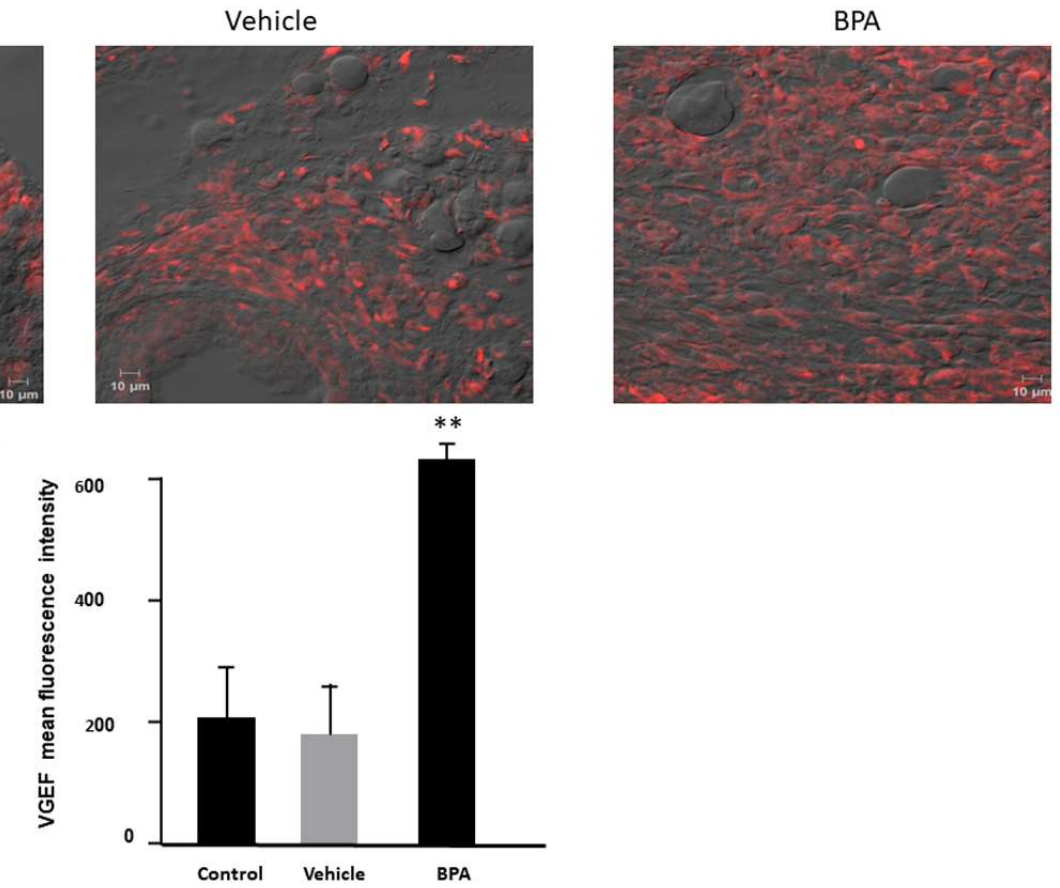

Figure 7. Intratumoral expression of VEGF. A. Representative images belonging to the intratumoral expression of VEGF
corresponding to the three experimental groups; control, vehicle, and BPA. B. Quantification of the expression of VEGF Each bar epresents the mean of a representative three different experiments ${ }^{* *}$ significant differences vs control and vehicle groups.

\subsection{Macro metastasis at the pulmonary level}

In addition to assessing the effect of BPA on tumor growth and due to the migration of cancer cells in mammary tumors that has tropism towards the lungs, we decided to take this organ to evaluate the phenomenon of metastasis. Figure 8 shows a comparison of the lungs of all experimental groups. We observed marked damage in the lungs of the BPA-treated animals compared to the control and vehicle-treated groups, which seems to collapse. In the image, it is not clear, but there are macroscopic lesions on the surface of the lung. It is important to note that at this time of sacrifice, even when the lungs were very damaged, the animals did not show apparent signs of pain or suffering.
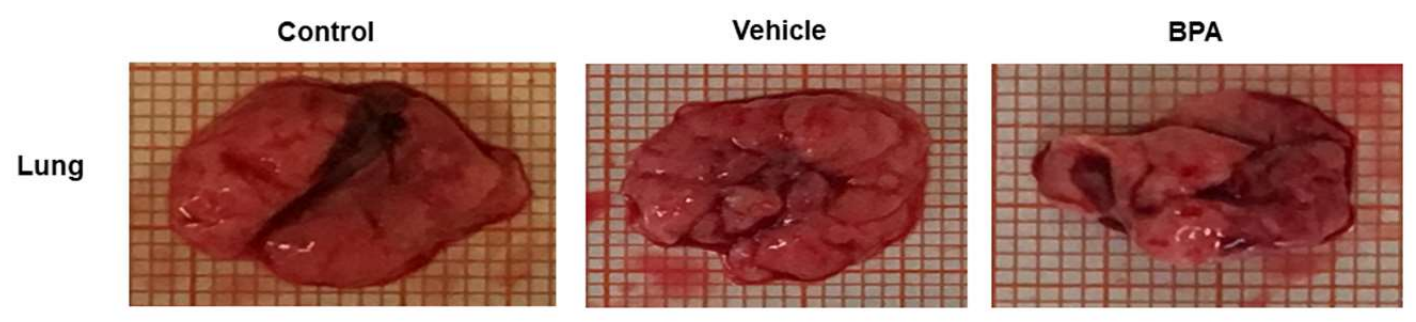

Figure 8. Macro metastasis at the pulmonary level. Representative images of the macro metastasis (tissue lessons) identified in the lungs belonging to control, vehicle and BPA-treated groups; millimetric grid as background.

\subsection{Histological examination of lungs in normal animals without tumors}

Then we decided to explore the histological damage of the lungs due to exposure to $\mathrm{BPA}$ in female mice without tumors. Figure 9 shows the comparison of the 
INTACT LUNG

VEHICLE

INTACT LUNG

$4 \mathrm{x}$
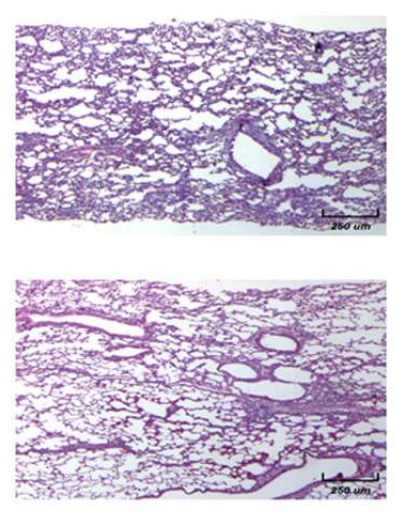

BPA INTACT LUNG microarchitecture of the lungs among experimental groups, control, vehicle and BPA treated animals at different magnifications $(4 \mathrm{x}, 10 \mathrm{x}$, and $40 \mathrm{x})$. It can be seen that there is no inflammation, not necrotic foci, and no new vascularization in any of the representative images shown. Of note, BPA treatment induced a slight inflammatory infiltrate into the lungs, as was judged in the 40x magnification in the center of the image. Moreover, the animals have no signs of disease (fur appearance, motility, mobility, food and water intake, and other behaviors were similar among nontumor groups).
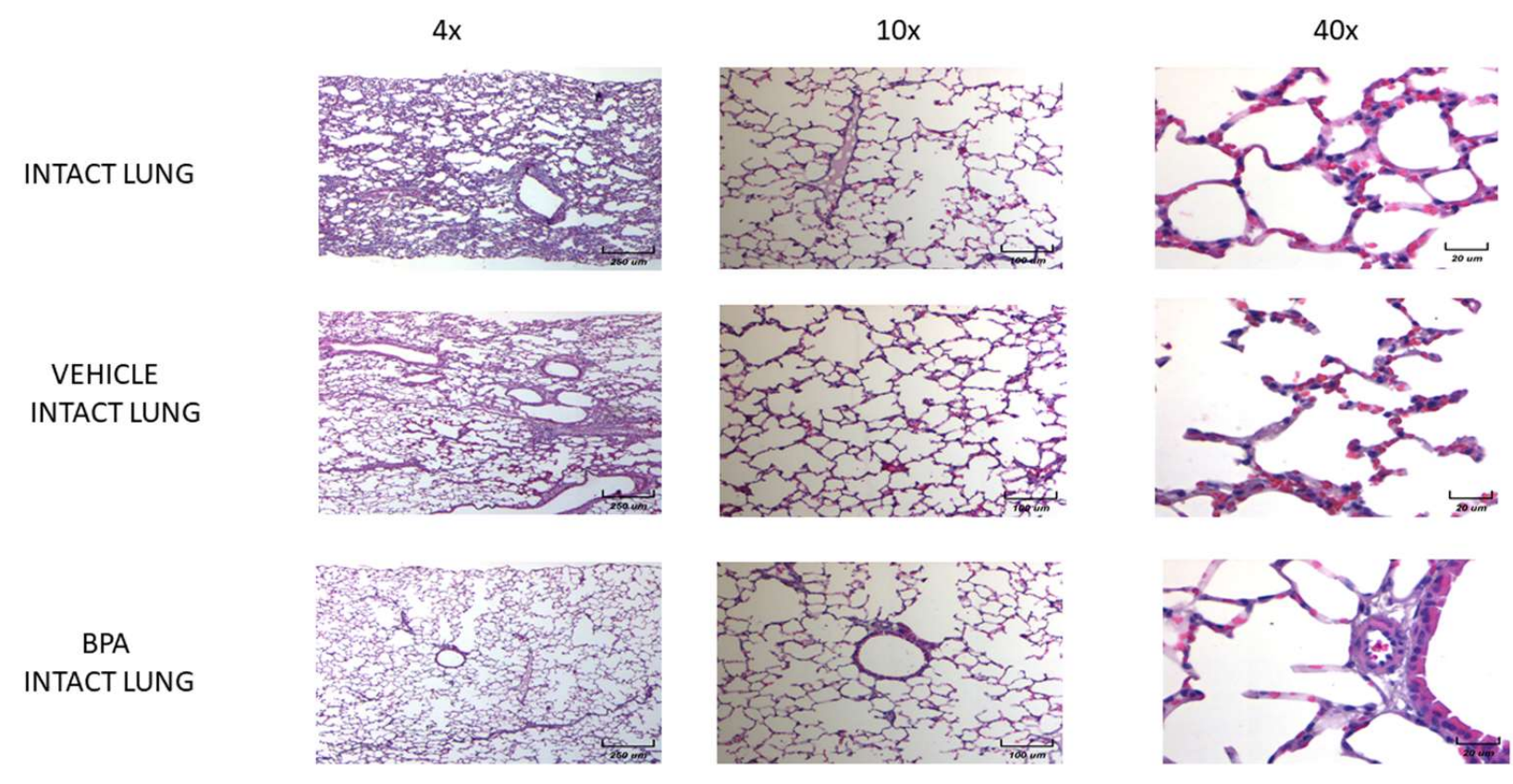

Figure 9. Histological examination of the lungs of female mice without tumors. Representative images of the lungs of female mice without tumor induction neonatally exposed to BPA at different magnifications (4x, 10x, and $40 \mathrm{x}$ ).

\subsection{Histological examination of lungs and micro metastases}

Micrometastases of control and vehicle animals are shown at the pulmonary level at the same magnifications as previously mentioned to analyze the morphology and cellular infiltrate. Usually multiple, bilateral, sharply outlined, rapidly growing, more pleomorphic, and necrotic sites were observed in both groups. The lung metastases were generally multiple, well-circumscribed and tend to grow rapidly. They appeared as multiple discrete nodules in the periphery of the lungs or as lymphangitic carcinomatosis (peribronchial and perivascular patterns via the lymphatics). Rarely appear as intralymphatic microscopic foci that cause pulmonary hypertension (Figure 10). 
INTACT 4T1
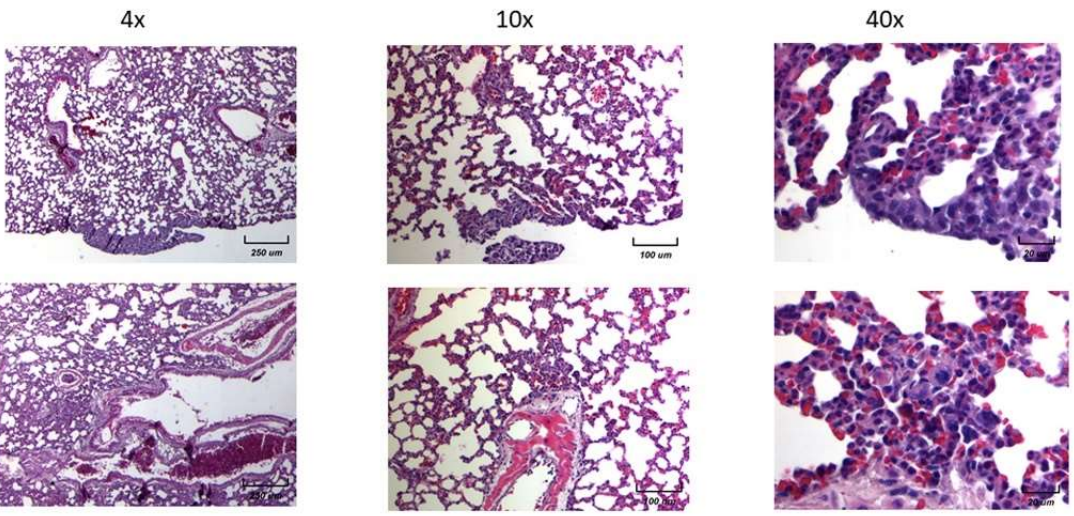

Figure 10. Histological examination of the lungs of female mice exposed to BPA without tumors. Representative images of the lungs in female mice exposed to BPA without tumor induction at different magnifications $(4 \mathrm{x}, 10 \mathrm{x}$, and $40 \mathrm{x}) .5$ animals were used for each experimental condition. Corroborar si se utilizaron este número de animales.

\subsection{Histological examination of the BPA-treated animals with tumors}

The results showed that the animals exposed to BPA had severe histological changes. For example, we observed an alveolar collapse. It should be noted that the alveoli were significantly infiltrated with neutrophils at the arterial level (center) and in medium vessels (arrows). The above can correlate with the air spaces (right) observed. On the other hand, the parenchyma had less density of neutrophils, which apparently present good alveolar ventilation. The bronchioles (star) show focal epithelial detachment. The number of micrometastasis and neutrophils in the lung parenchyma was higher in animals exposed to BPA than in the control and vehicle groups. Photomicrographs are higher magnifications of the metastases (40x). In this series of photomicrographs, several micrometastatic sections were observed, some at higher magnifications. Furthermore, the images showed increased subpleural micrometastasis, with a high presence of neutrophils and macrophages (Figure 11).
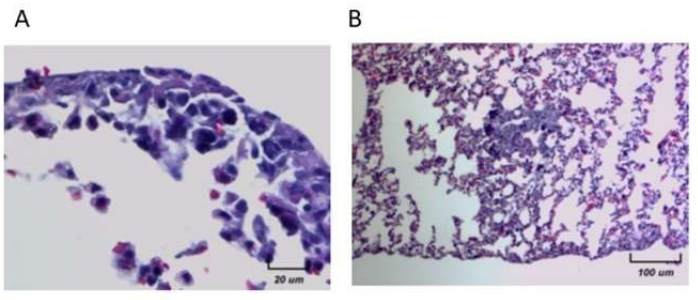

c

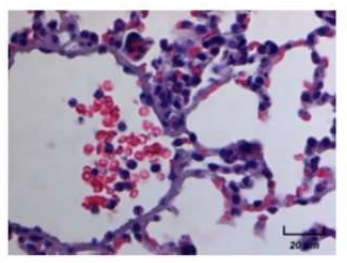

D

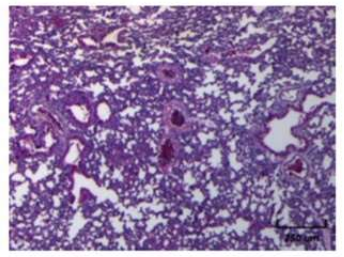

E

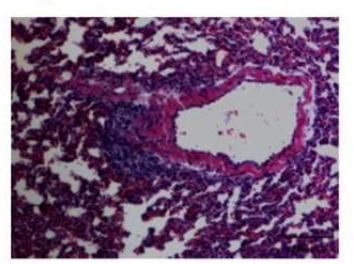

$\mathrm{F}$

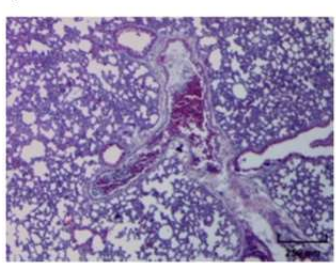

G

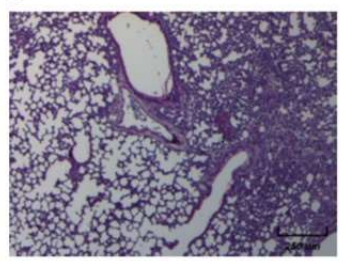

Figure 11. Histological examination of the BPA-treated animals with tumors. Representative images of the lungs in female mice neonatally exposed to BPA with a tumor induction onset the puberty. The images are displayed at different magnifications $(4 x, 10 x$, and 40x). 5 animals were used for each experimental condition. Corroborar si se utilizaron este número de animales . 


\section{Discussion}

In the present work, we showed for the first time that a single neonatal administration of BPA induces higher histological alterations at the pulmonary level, that were associated with important changes in the intratumoral expression of cytokines. As has been widely reported, the metastatic process can be promoted by the systemic and intratumoral production of cytokines [30-32]. For that reason, in a female mammary tumor model, we studied whether BPA treatment could affect intratumoral expression of IL-1 $\beta$, IL-4, IL-6, IL-10, TNF- $\alpha$, IFN-g and VGEF by confocal microscopy immunofluorescence.

The effects of BPA on immune system cells have been reported on the immune system cells; however, they vary depending on the model performed [33]. In vivo, the effects reported may seem contradictory, but they vary due to the animal species used, the dose, the administration route, the sex of the animal, the age, and the animal's development stage in which BPA is administered [34, 35]. Furthermore, many reports do not employ immune challenges for different immune components. Furthermore, there is little information on the effects of BPA on the immune response during cancer context [34]. Most of the BPA effects on the immune system, point out to BPA as a proinflammatory molecule [36-38]. Our results support and extend this notion, since the neonatal treatment of BPA clearly increased the pro-inflammatory cytokine profile.

Of particular interest is the finding that BPA decreases intratumoral expression of IL4. IL-4 is a cytokine produced mainly by innate immune system cells and Th2 cells, it plays an important role in the humoral immune response against parasites and allergic antigens [39]. However, it has also been shown to be involved in tumor growth, mediating increased proliferation and survival by promoting tumor-associated macrophage differentiation (TAM) towards an M2-like phenotype [40]. Our results do not agree with the role of IL-4 as a prometastasic molecule. However, it must be said that it is not the only cytokine that promotes metastasis, and that not all molecular pathways may be activated in every context. In our present work, IL-4 appears to be not as relevant as the other cytokines found. Interestingly, we observed that the expression of IL-1 $\beta$ was higher in the BPA-treated group. IL-1 $\beta$ is a pleiotropic cytokine involved in inflammatory processes $[41,42]$. In different studies, elevated levels of this cytokine have been observed in BC tumors and it has been proposed as a factor that promotes metastasis [43, 44]. Thus, our present results confirm and extend the pro-metastatic role of this cytokine. Like IL-1 $\beta$, IL6 is also considered a master pro-inflammatory cytokine [45]. Interestingly, we found a higher intratumoral induction of IL- 6 by neonatal exposure to BPA. In connection with this, in BC, several studies have shown a positive relationship between serum levels of IL6 and the progression of the disease. In fact, the elevated concentration of this cytokine has been considered as a negative marker of prognosis in BC, independently of many factors, including hormonal status [46]. Particularly, IL-6 can promote metastasis by aberrantly activating the STAT3 pathway, supporting cancer stem cells (CSC) [47]. The activation of the IL-6/JAK/STAT3 pathway has been implicated in the progression of BC [48]. Another pro-inflammatory molecule is TNF- $\alpha$, which is a multifunctional proinflammatory cytokine that regulates different processes, such as inflammation, cell apoptosis, tumor growth, and cell invasion [49]. We also found an increased intratumoral expression of this cytokine after neonatal exposure to BPA. Regarding cancer, TNF- $\alpha$ promotes invasion of breast tumor cells, as evidenced by in vitro experiments, upregulating several genes that are associated with proliferation, invasion, and metastasis $[50,51]$. Also, it has been shown that TNF- $\alpha$ can modulate the inflammatory role of macrophages, enhancing the production of VEGF [52]. Regarding BC metastasis, it has been well established that a critical regulator of this process is the VEGF family, which is shaped by various receptors and ligands that confer a poor prognosis to patients with BC [53]. Specifically, VEGF-A has been closely related to neovascularization and angiogenesis in BC cells $[54,55]$. It is important to note that we did not analyze different types of VEGF in the tumor; however, we observed that the expression of VEGF-A was outstandingly modulated by neonatal exposure to BPA, since it has a three-fold increase in response to 
it as compared to the other experimental conditions. Our results on the implications of VEGF and metastasis are related to recent reports carried out in a BC lung metastasis mice model.

This work demonstrated an important relationship between VEGFR and metastasisassociated macrophages (MAMs), proving that this population is firmly implicated in the tropism of lung metastasis in this disease $[56,57]$. This agrees with our previous work, in which neonatal exposure to BPA modulated macrophage genes, which are involved in the promotion of the alternative phenotype with important implications in metastasis. In addition, the stimulating role of VEGF by different disrupting compounds, including $\mathrm{BPA}$, have also been recently reported in BC cells [6, 7].

In addition to the above, a weakness of this work is that we did not evaluate mechanisms by which BPA can modulate the expression of cytokines. Nevertheless, it has been reported that this endocrine disruptor, through its binding to the membrane ER, can impact in the expression of various transcription factors including PPAR $\gamma$ which has pleiotropic actions at multiple levels and is importantly involved in the functioning of immune cells $[58,59]$. Different studies that investigate the mechanism through BPA can modulate the expression of cytokines would be carried out to expand this information.

What it is interesting, it is the fact that we found an angiogenic/immunological modulation driven by BPA, which invites us to extend the cancer therapy options not only to breast cancer cells and cytokine signaling, but also to their endocrine disruptor counterpart.

\section{Conclusions}

Metastasis is the major cancer pathophysiology and there are multiple biological interactions that converge in common signaling pathways. The knowledge of this network leads to the possibility of developing specific therapeutic target drugs. Endocrinedisrupting compounds modulate endogenous hormone responses and cell functions. Although most studies have focused on their reproductive effects, their potential effects on immune cells and even more, on the immune response towards cancer, should draw attention, given the expression of hormonal receptors by immune cells. Even though a lot of studies have evaluated the effects of BPA in the immune response. However, more studies are needed with the aim to elucidate the possible mechanisms by which these take place. In this here we exposed different molecular targets that should be blocked together, with the aim to offer a promising anti-metastatic drug regardless of the expression of hormonal receptors. We also want to highlight that not only cancer cells must be considered but also the modification of the tumor microenvironment and the metabolism of the surrounding cells play a key role in attacking the metastatic BC process and it would result in a better patient clinical outcome. In addition, the immune cells and cytokines are key factors whose modulation would be important as adjuvant drug options in breast cancer metastasis.

Supplementary Materials: Supplementary figure 1. Immunofluorescence negative controls of the different stains.

Author Contributions: Conceptualization, JMM, KENC; Methodology, MIPA, NAMM, APT, KENC. Validation, MIPA, KENC Formal analysis, JMM Resources, JMM; Writing-original draft preparation, MIPA, MSM, JMM.; Writing-review and editing, JMM, MIPA, MSM; Supervision, JMM, Funding acquisition, JMM. All authors have read and agreed to the published version of the manuscript.

Funding: This research was funded by Funding by Grant IN-209719 from Programa de Apoyo a Proyectos de Innovación Tecnológica (PAPIIT), Dirección General de Asuntos del Personal Académico (DGAPA), Universidad Nacional Autónoma de México (UNAM) and Grant FC20162125 from Fronteras en la Ciencia, Consejo Nacional de Ciencia y Tecnología (CONACYT), both to Jorge Morales-Montor.

Institutional Review Board Statement: All experimental procedures in the animals were approved by the Institutional Care and Animal Use Committee (CICUAL), permit number 155, adhering to 
Mexican regulation (NOM-062-ZOO-1999), and in accordance with the recommendations from the National Institute of Health (NIH) of the United States of America (Guide for the Care and Use of Laboratory Animals).

Data Availability Statement: The datasets generated and analyzed during the current study are included in the present manuscript. Also, they are available from the corresponding author on request.

Conflicts of Interest: The authors declare no conflict of interest.

\section{References}

1. Sung, H., et al., Global Cancer Statistics 2020: GLOBOCAN Estimates of Incidence and Mortality Worldwide for 36 Cancers in 185 Countries. CA Cancer J Clin, 2021. 71(3): p. 209-249.

2. Metzger-Filho, O., et al., Mixed Invasive Ductal and Lobular Carcinoma of the Breast: Prognosis and the Importance of Histologic Grade. The oncologist, 2019. 24(7): p. e441-e449.

3. Arps, D.P., et al., Invasive ductal carcinoma with lobular features: a comparison study to invasive ductal and invasive lobular carcinomas of the breast. Breast cancer research and treatment, 2013. 138(3): p. 719-726.

4. Fares, J., et al., Molecular principles of metastasis: a hallmark of cancer revisited. Signal Transduction and Targeted Therapy, 2020. 5(1): p. 28.

5. Mendez-Garcia, L.A., et al., Breast Cancer Metastasis: Are Cytokines Important Players During Its Development and Progression? J Interferon Cytokine Res, 2019. 39(1): p. 39-55.

6. Buteau-Lozano, H., et al., Xenoestrogens modulate vascular endothelial growth factor secretion in breast cancer cells through an estrogen receptor-dependent mechanism. J Endocrinol, 2008. 196(2): p. 399-412.

7. Segovia-Mendoza, M., et al., The chemical environmental pollutants BPA and BPS induce alterations of the proteomic profile of different phenotypes of human breast cancer cells: A proposed interactome. Environ Res, 2020. 191: p. 109960.

8. Soto, A.M. and C. Sonnenschein, Environmental causes of cancer: endocrine disruptors as carcinogens. Nature reviews. Endocrinology, 2010. 6(7): p. 363-370.

9. Segovia-Mendoza, M., et al., How microplastic components influence the immune system and impact on children health: Focus on cancer. Birth Defects Res, 2020. 112(17): p. 1341-1361.

10. Dunn, G.P., L.J. Old, and R.D. Schreiber, The immunobiology of cancer immunosurveillance and immunoediting. Immunity, 2004. 21(2): p. 137-48.

11. Smyth, M.J., G.P. Dunn, and R.D. Schreiber, Cancer immunosurveillance and immunoediting: the roles of immunity in suppressing tumor development and shaping tumor immunogenicity. Adv Immunol, 2006. 90: p. 1-50.

12. Eelen, G., et al., Basic and Therapeutic Aspects of Angiogenesis Updated. Circ Res, 2020. 127(2): p. 310-329.

13. Potente, M., H. Gerhardt, and P. Carmeliet, Basic and therapeutic aspects of angiogenesis. Cell, 2011. 146(6): p. 873-87.

14. Al-Rawi, M.A. and W.G. Jiang, Lymphangiogenesis and cancer metastasis. Front Biosci (Landmark Ed), 2011. 16: p. 723-39.

15. Klagsbrun, M. and P.A. D'Amore, Vascular endothelial growth factor and its receptors. Cytokine Growth Factor Rev, 1996. 7(3): p. 259-70.

16. Drevs, J., VEGF and angiogenesis: implications for breast cancer therapy. European Journal of Cancer Supplements, 2008. 6(6): p. 7-13.

17. Le, H.H., et al., Bisphenol A is released from polycarbonate drinking bottles and mimics the neurotoxic actions of estrogen in developing cerebellar neurons. Toxicology letters, 2008. 176(2): p. 149-156.

18. Almeida, S., et al., Bisphenol A: Food Exposure and Impact on Human Health. Compr Rev Food Sci Food Saf, 2018. 17(6): p. 1503-1517.

19. Goodson, A., et al., Migration of bisphenol A from can coatings--effects of damage, storage conditions and heating. Food Addit Contam, 2004. 21(10): p. 1015-26.

20. Del Pup, L., et al., Carcinogenetic mechanisms of endocrine disruptors in female cancers (Review). Oncol Rep, 2016. 36(2): p. 603-12.

21. Cwiek-Ludwicka, K., Bisphenol A (BPA) in food contact materials - new scientific opinion from EFSA regarding public health risk. Rocz Panstw Zakl Hig, 2015. 66(4): p. 299-307.

22. Vandenberg, L.N., et al., Exposure to environmentally relevant doses of the xenoestrogen bisphenol-A alters development of the fetal mouse mammary gland. Endocrinology, 2007. 148(1): p. 116-27.

23. Ikezuki, Y., et al., Determination of bisphenol A concentrations in human biological fluids reveals significant early prenatal exposure. Hum Reprod, 2002. 17(11): p. 2839-41.

24. Vandenberg, L.N., et al., Urinary, circulating, and tissue biomonitoring studies indicate widespread exposure to bisphenol A. Cien Saude Colet, 2012. 17(2): p. 407-34.

25. Vandenberg, L.N., et al., Urinary, circulating, and tissue biomonitoring studies indicate widespread exposure to bisphenol A. Environ Health Perspect, 2010. 118(8): p. 1055-70.

26. Xu, J., G. Huang, and T.L. Guo, Developmental Bisphenol A Exposure Modulates Immune-Related Diseases. Toxics, 2016. 4(4).

27. Malaisé, Y., et al., Perinatal oral exposure to low doses of bisphenol A, S or F impairs immune functions at intestinal and systemic levels in female offspring mice. Environmental Health, 2020. 19(1): p. 93. 
28. Ratajczak-Wrona, W., et al., Effect of bisphenol A on human neutrophils immunophenotype. Scientific Reports, 2020. 10(1): p. 3083.

29. Palacios-Arreola, M.I., et al., A single neonatal administration of Bisphenol A induces higher tumour weight associated to changes in tumour microenvironment in the adulthood. Scientific Reports, 2017. 7(1): p. 10573-10573.

30. Esquivel-Velázquez, M., et al., The role of cytokines in breast cancer development and progression. J Interferon Cytokine Res, 2015. 35(1): p. 1-16.

31. Kawaguchi, K., et al., Alteration of specific cytokine expression patterns in patients with breast cancer. Scientific Reports, 2019. 9(1): p. 2924.

32. Kim, Y.W., et al., Association of serum and intratumoral cytokine profiles with tumor stage and neutrophil lymphocyte ratio in colorectal cancer. Anticancer Res, 2014. 34(7): p. 3481-7.

33. Youn, J.Y., et al., Evaluation of the immune response following exposure of mice to bisphenol A: Induction of th1 cytokine and prolactin by BPA exposure in the mouse spleen cells. Archives of Pharmacal Research, 2002. 25(6): p. 946.

34. Araiza, V., et al., Bisphenol A, an endocrine-disruptor compund, that modulates the immune response to infections. Front Biosci (Landmark Ed), 2021. 26: p. 346-362.

35. Richter, C.A., et al., In vivo effects of bisphenol A in laboratory rodent studies. Reproductive toxicology (Elmsford, N.Y.), 2007. 24(2): p. 199-224.

36. Savastano, S., et al., Bisphenol-A plasma levels are related to inflammatory markers, visceral obesity and insulin-resistance: a cross-sectional study on adult male population. Journal of translational medicine, 2015. 13: p. 169-169.

37. Chen, Y., H.S. Xu, and T.L. Guo, Modulation of cytokine/chemokine production in human macrophages by bisphenol A: A comparison to analogues and interactions with genistein. J Immunotoxicol, 2018. 15(1): p. 96-103.

38. Liu, Y., et al., Modulation of cytokine expression in human macrophages by endocrine-disrupting chemical Bisphenol-A. Biochem Biophys Res Commun, 2014. 451(4): p. 592-8.

39. Bao, K. and R.L. Reinhardt, The differential expression of IL-4 and IL-13 and its impact on type-2 immunity. Cytokine, 2015. 75(1): p. 25-37.

40. Wang, H.-W. and J.A. Joyce, Alternative activation of tumor-associated macrophages by IL-4. Cell Cycle, 2010. 9(24): p. 48244835.

41. Eskan, M.A., et al., Interleukin-1beta modulates proinflammatory cytokine production in human epithelial cells. Infect Immun, 2008. 76(5): p. 2080-9.

42. Dinarello, C.A., Biologic basis for interleukin-1 in disease. Blood, 1996. 87(6): p. 2095-147.

43. Tulotta, C. and P. Ottewell, The role of IL-1B in breast cancer bone metastasis. Endocrine-related cancer, 2018. 25(7): p. R421R434.

44. Tulotta, C., et al., Endogenous Production of IL1B by Breast Cancer Cells Drives Metastasis and Colonization of the Bone Microenvironment. Clin Cancer Res, 2019. 25(9): p. 2769-2782.

45. Tanaka, T., M. Narazaki, and T. Kishimoto, IL-6 in inflammation, immunity, and disease. Cold Spring Harbor perspectives in biology, 2014. 6(10): p. a016295-a016295.

46. Salgado, R., et al., Circulating interleukin-6 predicts survival in patients with metastatic breast cancer. Int J Cancer, 2003. 103(5): p. 642-6.

47. Zhang, C., K. Ma, and W.Y. Li, IL-6 Promotes Cancer Stemness and Oncogenicity in U2OS and MG-63 Osteosarcoma Cells by Upregulating the OPN-STAT3 Pathway. J Cancer, 2019. 10(26): p. 6511-6525.

48. Johnson, D.E., R.A. O'Keefe, and J.R. Grandis, Targeting the IL-6/JAK/STAT3 signalling axis in cancer. Nature reviews. Clinical oncology, 2018. 15(4): p. 234-248.

49. Kobelt, D., et al., Pro-inflammatory TNF-alpha and IFN-gamma Promote Tumor Growth and Metastasis via Induction of MACC1. Front Immunol, 2020. 11: p. 980.

50. Semesiuk, N.I., et al., Disseminated tumor cells and enhanced level of some cytokines in bone marrow and peripheral blood of breast cancer patients as predictive factors of tumor progression. Exp Oncol, 2013. 35(4): p. 295-302.

51. Wolczyk, D., et al., TNF-alpha promotes breast cancer cell migration and enhances the concentration of membrane-associated proteases in lipid rafts. Cell Oncol (Dordr), 2016. 39(4): p. 353-63.

52. Lu, P., et al., Critical role of TNF-alpha-induced macrophage VEGF and iNOS production in the experimental corneal neovascularization. Invest Ophthalmol Vis Sci, 2012. 53(7): p. 3516-26.

53. Su, J.L., et al., The VEGF-C/Flt-4 axis promotes invasion and metastasis of cancer cells. Cancer Cell, 2006. 9(3): p. $209-23$.

54. Ferrara, N. and T. Davis-Smyth, The biology of vascular endothelial growth factor. Endocr Rev, 1997. 18(1): p. 4-25.

55. Zhang, J., et al., Suppression of breast cancer metastasis through the inhibition of VEGF-mediated tumor angiogenesis. Cancer therapy, 2007. 5: p. 273-286.

56. Qian, B.Z., et al., FLT1 signaling in metastasis-associated macrophages activates an inflammatory signature that promotes breast cancer metastasis. J Exp Med, 2015. 212(9): p. 1433-48.

57. Qian, B., et al., A distinct macrophage population mediates metastatic breast cancer cell extravasation, establishment and growth. PLoS One, 2009. 4(8): p. e6562.

58. Cimmino, I., et al., Potential Mechanisms of Bisphenol A (BPA) Contributing to Human Disease. International journal of molecular sciences, 2020. 21(16): p. 5761.

59. Zhang, X. and H.A. Young, PPAR and immune system--what do we know? International immunopharmacology, 2002. 2(8): p. 1029-1044. 
